\section{e0616 THE PREDICTION VALUE OF BNP NTPROBNP AND THEIR RATIO FOR INHOSPITAL OUTCOMES IN CONGESTIVE HEART FAILURE}

doi:10.1136/hrt.2010.208967.616

${ }^{1}$ Dai Yuxiang, ${ }^{2}$ Konishi Hakuoh, ${ }^{2}$ Takagi Atsutoshi, ${ }^{2}$ Miyauchi Katsumi, ${ }^{2}$ Daida Hiroyuki. ${ }^{1}$ Fudan University Affiliated Zhongshan Hospital, China; ${ }^{2}$ Juntendo University, Japan

Objectives BNP and NT-proBNP are important cardiac biomarkers in the diagnosis and prognosis of congestive heart failure (CHF). However, the prognosis value of their ratio in patients with CHF is not clear. The goal of this study was to examine the prediction value of BNP, NT-proBNP and their ratio for in-hospital outcomes in CHF. Methods In a cross-sectional study, patients with acute onset of CHF and admitted to cardiac care unit in Juntendo Hospital were enrolled from Jan to Dec 2009. We measured the serum level of BNP and NT-proBNP at the same time after admission, and other biomarkers were also measured and collected. The results were statistically analysed by software JMP 7.

Results A total of 193 patients were enrolled, with a mean age of $71.3 \pm 12.8$ years old. 17 patients died in hospital, with a mortality rate of $8.8 \%$. Univariate analysis showed that in-hospital mortality was significantly related with BMI, BNP, NT-proBNP, the ratio of NT-proBNP/BNP, RDW, LDL-C and CRP. The mean ratio of NTproBNP/BNP was $16.7 \pm 11.6$ for in-hospital death group and $9.5 \pm 8.6$ for in-hospital relieving group $(p<0.05)$. Logistic and multiple regression analysis showed that the ratio of NT-proBNP/ $\mathrm{BNP}$ was an independent predictor for both in-hospital mortality and duration in hospital.

Conclusions The ratio of NT-proBNP/BNP is better for predicting in-hospital outcomes than BNP or NT-proBNP in congestive heart failure and it might be useful to predict short term outcomes in patients with acute exacerbation of heart failure.

\section{e0617 RESEARCH OF RELATION BETWEEN THE TYPE OF LEFT VENTRICULAR REMODELLING AND ATRIAL FIBRILLATION IN PATIENTS WITH ESSENTIAL HYPERTENSION}

doi:10.1136/hrt.2010.208967.617

${ }^{1}$ Li Zhi, ${ }^{2}$ Lu Xilie, 'Zhu Jinxiu, ${ }^{1}$ Wang Jianfeng. 'First Affiliated Hospital of Shantou University Medical College; ${ }^{2}$ The General Hospital of Pla

Background Essential hypertension (EH) is the common cause of left ventricular (LV) geometric remodelling which includes shape remodelling, volume remodelling and mass remodelling. The relation between the type of LV geometric remodelling and atrial fibrillation (AF) in patients with $\mathrm{EH}$ was unknown.

Objectives To explore the relation between the type of left ventricular geometric remodelling and $\mathrm{AF}$ in patient with $\mathrm{EH}$.

Methods In accordance with hypertension guideline (JNC-7), consecutive inpatients with $\mathrm{EH}$ ( $\mathrm{n}=211$, from September, 2008 to August, 2009) were enrolled at the department of cardiology in PLA general hospital. The patients were divided into AF group and sinus rhythm (SR) group by baseline heart rhythm. The diagnosis of AF was conformed to ACC/AHA/ESC 2006 guidelines for the management of patients with AF executive summary. The patients underwent Doppler echocardiography examination which included LV sphericity index (LVSI), LV diastolic volume (LVEDV), LV mass (LVM) and left atrial sphericity index (LASI). The clinical and echocardiographic characteristics were compared by t test, Chisquare test and multiple Logistic regression analysis. Then AF group was divided into persistent group and paraxysmal group. The indices of echocardiography among three groups were compared by analysis of variance.
Results Compared to SR group, AF group has lower systolic blood pressure and diastolic blood pressure $(138.85 \pm 19.89 \mathrm{~mm} \mathrm{Hg}$ vs $149.80 \pm 25.17 \mathrm{~mm} \mathrm{Hg} ; 80.57 \pm 13.04 \mathrm{~mm} \mathrm{Hg}$ vs $85.97 \pm 15.81 \mathrm{~mm}$ $\mathrm{Hg}, \mathrm{p}<0.01)$ and larger LVM, LVEDV, LVESV and LVSI $(108.19 \pm 27.52 \mathrm{~g}$ vs $99.69 \pm 21.61 \mathrm{~g}, \quad \mathrm{p}<0.05 ; 199.20 \pm 57.00 \mathrm{ml}$ vs $181.92 \pm 50.62 \mathrm{ml}, \quad \mathrm{p}<0.05 ; \quad 45.52 \pm 20.03 \mathrm{ml}$ vs $37.60 \pm 13.03 \mathrm{ml}$, $\mathrm{p}<0.01 ; 0.715 \pm 0.043$ vs $0.688 \pm 0.040, \mathrm{p}<0.01)$, although LASI was significantly smaller $(0.740 \pm 0.081$ vs $0.779 \pm 0.08, p<0.01)$. Given covariates were adjusted in the logistic regression model, the LVSI, $\mathrm{LAD}$ and LASI were independent factors associated with AF in patients with hypertension (OR:0.847, 95\% CI 6.141 138.590 , $\mathrm{p}<0.01$; OR: 0.811, 95\% CI 0.738 $\pm 0.891, \mathrm{p}<0.01$; OR: $65.836,95 \% \mathrm{CI}$ $0.764 \pm 0.942, p<0.01)$

Conclusions Compared to SR group, AF group has significant variation in the type of left ventricular geometric remodelling in $\mathrm{EH}$ patients. The LVM and LV volume are much larger and there is a sphericity trend of LV in EH patients with AF. However, there is a deviation of sphericity of left atrial in $\mathrm{EH}$ patients with AF. The LVSI, LAD and LASI were the independent risk factors of $\mathrm{EH}$ patients with AF after adjustment for other covariates. With the development of AF, the degree of left ventricular geometric remodelling is increasing.

\section{e0618 EFFECTS OF ROSUVASTATIN ON PLASMA NO AND ET-1 DURING MYOCARDIAL ISCHAEMIA-REPERFUSION INJURY IN RABBITS}

doi:10.1136/hrt.2010.208967.618

${ }^{1}$ MA Jian-qun, ${ }^{1}$ ZHANG Lei, ${ }^{2}$ WANG Lian-wen, 'ZHU Yu-qin, 'ZHU Yu-hong. ${ }^{1}$ Department of Cardiology, the Affiliated Hospital of Binzhou Medical College; ${ }^{2}$ Department of nuclear medicine, the Affiliated Hospital of Binzhou Medical College Binzhou, China

Objective To study the change of endothelial function during myocardial ischaemia-reperfusion injury in rabbits and the effect of Rosuvastatin.

Methods $16 \mathrm{New}$ Zealand rabbits were randomLy divided into two groups: ischaemia/reperfusion injury group (control group) and Rosuvastatin group (drug group). Establish the myocardial ischaemia-reperfusion model. The camponotus upward elevation $(\geq 0.2 \mathrm{mv})$ of the ST segment shown by the ECG indicated the successful ligation of the left anterior descending coronary artery; 40 mins later the ligation line was cut off, and the ST segment of ECG returned to $1 / 2$ or more, which showed the success of reperfusion. At the four time points, before occlusion, $40 \mathrm{~min}$ after occlusion, 60 mins and 180 mins after reperfusion. We measured rabbit serum nitric oxide (NO), plasma endothelia-1 (ET-1) content. SPSS 11.5 software was applied, using ANOVA to $p<0.05$ for differences with statistical significance.

Results In both groups, the serum NO content reduced gradually and the plasma ET-1 content increased gradually with protraction of the ischaemia and reperfusion time. Before ischaemia the serum NO $[(109.875 \pm 32.255) \mu \mathrm{mol} / \mathrm{l}$ vs $(114.500 \pm 37.405) \mu \mathrm{mol} / \mathrm{l}, \mathrm{p}>0.05]$ and plasma ET-1 $[(221.111 \pm 28.125) \mathrm{pg} / \mathrm{ml}$ vs $(204.594 \pm 31.790) \mathrm{pg} / \mathrm{ml}$, $\mathrm{p}>0.05]$, have no significant difference between the groups. At other three time points, the increased serum NO content [(63.125 \pm 18.962$)$,

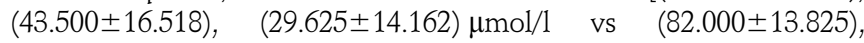
(63.375 \pm 17.541$), \quad(50.250 \pm 18.987) \mu \mathrm{mol} / \mathrm{l}, \mathrm{p}<0.05]$ in drug group was markedly lower than that in the control group and the reduced plasma ET-1 content [(331.785 \pm 35.341$),(375.914 \pm 45.204)$, $(459.829 \pm 70.110) \mathrm{pg} / \mathrm{ml}$ vs $(282.541 \pm 38.928), \quad(315.152 \pm 55.263)$, $(377.795 \pm 60.427) \mathrm{pg} / \mathrm{ml}, \mathrm{p}<0.05]$ in drug group was markedly higher than those in the control group.

Conclusions Through increasing serum NO, and reducing plasma ET-1, rosuvastatin can improve endothelial function in myocardial ischaemia-reperfusion injury rabbits. 